Borneo Journal of Sciences \& Technology, 4(1): 65-69

DOI: http://doi.org/10.3570/bjost.2022.4.1-10

e-ISSN: 2672-7439

(C) 2018, UTS Publisher.

Submitted: $30^{\text {th }}$ November 2021

Accepted: $05^{\text {th }}$ January 2022

Published: $31^{\text {st }}$ January 2022

\title{
Sensorial Characteristics of Noodles Incorporated with Local Dabai Fruit (Canarium odontophyllum) Powder
}

\author{
${ }^{* 1,2}$ Farah Syahirah Abdul Shukri, ${ }^{2}$ Yii Hung Wei, ${ }^{1,2}$ Mohd Zahid Abidin, ${ }^{3}$ Norhashila Hashim, \\ ${ }^{3}$ Mohd Nazren Radzuan, ${ }^{4}$ Mohd Sabri Pak Dek
}

\author{
${ }^{1}$ Centre for Research of Innovation and Sustainable Development (CRISD), University of Technology \\ Sarawak, 96000 Sibu, Sarawak, Malaysia \\ ${ }^{2}$ School of Engineering and Technology, University of Technology Sarawak, \\ 96000 Sibu, Sarawak, Malaysia \\ ${ }^{3}$ Department of Biological \& Agricultural Engineering, Faculty of Engineering, Universiti Putra Malaysia, \\ 43400 Serdang, Selangor, Malaysia \\ ${ }^{4}$ Department of Food Science, Faculty of Food Science \& Technology, Universiti Putra Malaysia, 43400 \\ Serdang, Selangor, Malaysia
}

\begin{abstract}
Noodles are different from pasta and can be differentiated based on their ingredients and manufacturing processes. Noodles are made from common wheat flour and have high popularity among the Asian countries. The objective of this study is to evaluate the sensory properties of noodles incorporated with local Dabai fruit (Canarium odontophyllum) powder. In this study, noodles were prepared with three different level percentages $(10 \%, 20 \%$, and $30 \%$ ) of Dabai powder as wheat flour substitution. The four-formulation including a control were evaluated for the sensory properties in terms of colour, aroma, taste, texture, overall acceptability and purchase intention. Among all the formulations, Dabai noodles of formulation 1 (10\% Dabai) showed significant overall preference by the panelists. Panelists found that noodles incorporated with 10\% Dabai powder are attractive for normal consumer while noodles incorporated with 30\% Dabai powder are appealing for Dabai fruits lovers considering the aroma and taste as well.
\end{abstract}

Keywords: Dabai, Dabai powder, noodles, sensory evaluation.

\section{INTRODUCTION}

Noodles are one of the staple foods famous in Asia and have a history of 4000 years old. An overturned sealed bowl filled with long, thin, and preserved yellow noodles found in China explained the presence of noodles in the past. According to Roach [1], broomcorn millet and foxtail millet are liable for the production of noodles by comparing seed husks and starch grains with modern crops. Various types of noodles are found throughout Asia, and they are classified based on shapes, thickness gravy seasoning and compositions. [2].

The basic ingredients to make noodles are flour, eggs, salt and water. Types of flour chosen, addition of reducing and shortening agents and the composition of each ingredients effect the sensorial properties of noodles. There are a lot of compounds and methods used and applied to enrich the quality of noodles. Among them, apricot kernel flour, sweet potato, ragi, are used in recent noodles quality enrichment studies [3].

Wheat flour contain almost $80 \%$ of starch. There are six levels of structure of wheat starch, which are individual branches, whole molecules, semi-crystalline lamellae, growth rings, starch granules and finally grains. Glucose monomers with $\alpha(1 \rightarrow 4)$ glycosidic linkages and $\alpha(1 \rightarrow 6)$ glycosidic linkages are linked together with linear chains to form individual branches (level 1).

Amylopectin and amylose molecules, or amylopectin branches (level 2) are formed by linking chains of individual branches. Crystalline lamellae (level 3) is built from double helices formed by amylopectin branches. Semi crystalline growth rings (level 4) are formed in starch granule (level 5) by chains of repeating crystalline lamellae. In cell-well matrices and protein of wheat grains (level 6), starch granules are built along with lipids [4].

Dabai fruit is white in colour and it will turn to purplish black colour with an edible, thin skin when 
ripe. Three-angled pointed Dabai seed is covered with approximately $5 \mathrm{~mm}$ thick of yellow or white flesh [5].

Nutritional composition of Dabai fruits can be affected by climate, growing region, cultivar, maturity and cultural practice. By referring to Table 1, the Dabai fruits produced in Song and Sarikei have higher carbohydrate content when compared to Kapit and Kanowit. The Dabai fruit from Sarikei and Song have carbohydrate content of $9.16 \%$ and $8.97 \%$ respectively while Kanowit and Kapit have $4.45 \%$ and $5.07 \%$ respectively [6]. These unique composition of carbohydrate in Dabai fruit became the area of interest for sensorial characteristics of noodles enriched with underutilised Sarawak indigenous fruit Dabai (Canarium Odontophyllum) using different percentages of its powder as there are no scientific research conducted.

Table 1: Nutritional Composition of Dabai Fruits in Sarawak. Tan and Azlan [6]

\begin{tabular}{lccccc}
\hline $\begin{array}{l}\text { Nutritional } \\
\text { composition }\end{array}$ & $\begin{array}{l}\text { (Voon \& } \\
\text { Kueh, } \\
\text { 1999) }\end{array}$ & $\begin{array}{c}\text { Dabai } \\
\text { from } \\
\text { Sarikei }\end{array}$ & $\begin{array}{c}\text { Dabai } \\
\text { from } \\
\text { Song }\end{array}$ & $\begin{array}{c}\text { Dabai } \\
\text { from } \\
\text { Kapit }\end{array}$ & $\begin{array}{c}\text { Dabai } \\
\text { from } \\
\text { Kanowit }\end{array}$ \\
\hline Energy (kcal) & 339 & - & - & - & - \\
Fat (\%) & 26.2 & - & - & - & - \\
Protein (\%) & 3.8 & - & - & - & - \\
Moisture (\%) & 41.3 & & & & \\
Ash (\%) & 2.3 & & & & \\
Carbohydrates & 22.1 & 9.16 & 8.97 & 4.45 & 5.07 \\
(\%) & & - & - & - & - \\
Crude Fibre (\%) & 4.3 & & & & \\
Soluble Dietary & & & $0.0-0.16$ & & \\
Fibre (\%) & & & & & \\
Insoluble Dietary & & & & & \\
Fibre (\%) & & & & & \\
\hline
\end{tabular}

Note: “-” for not mentioned in that particular journal.

\section{MATERIALS AND METHODS}

\section{Materials}

Dabai Song was obtained from Pasar Sarikei. Dabai flesh and skin were peeled and dry in oven at $60{ }^{\circ} \mathrm{C}$ for 6 hours. Dried Dabai flesh and skin were ground and sieved using fine sieve with mesh size of $0.79 \mathrm{~mm}$ [7]. Nine hundred grams $(900 \mathrm{~g})$ of salt and $5 \mathrm{~kg}$ of wheat flour were purchase from Doremon Supermarket, Sarikei.

\section{Formulation and preparation of Dabai noodles}

Table 2: Formulation of Dabai Noodles

\begin{tabular}{lllll}
\hline \multirow{2}{*}{ Ingredients } & \multicolumn{4}{c}{ Samples } \\
\cline { 2 - 5 } & Control & $\mathbf{F}_{\mathbf{1}}$ & $\mathbf{F}_{\mathbf{2}}$ & $\mathbf{F}_{\mathbf{3}}$ \\
\hline Wheat flour $(\mathrm{g})$ & 100 & 90 & 80 & 70 \\
\hline Dabai powder $(\mathrm{g})$ & 0 & 10 & 20 & 30 \\
\hline Water $(\mathrm{ml})$ & 35 & 35 & 35 & 35 \\
\hline Salt $(\mathrm{g})$ & 1 & 1 & 1 & 1 \\
\hline
\end{tabular}

Note: $F_{1}=$ Formulation 1, $F_{2}=$ Formulation 2, $F_{3}=$ Formulation 3

Wheat flour content decreased from control to formulation $3(100 \mathrm{~g}>70 \mathrm{~g})$, Dabai powder content increased form control to formulation 3 (0 g > $30 \mathrm{~g}$ ) while the water $(35 \mathrm{ml})$ and salt $(1 \mathrm{~g})$ content remains the same. Then, Dabai powder and wheat flour were mixed with water and salt. The dough was allowed to rest for 30 minutes. The dough was rolled with rolling pin and extruded using a noodle extruder to obtained long and thick noodles.

Dabai noodles were steamed at $100{ }^{\circ} \mathrm{C}$ for 1 hour and 15 minutes. After steaming, Dabai noodles were dried at $60{ }^{\circ} \mathrm{C}$ for 30 minutes in a drying oven before vacuum packaged into polyethylene [8]. Vacuum packaged Dabai noodles were stored at room temperature on shelf. Figure 1 shows the preparation of Dabai noodles.

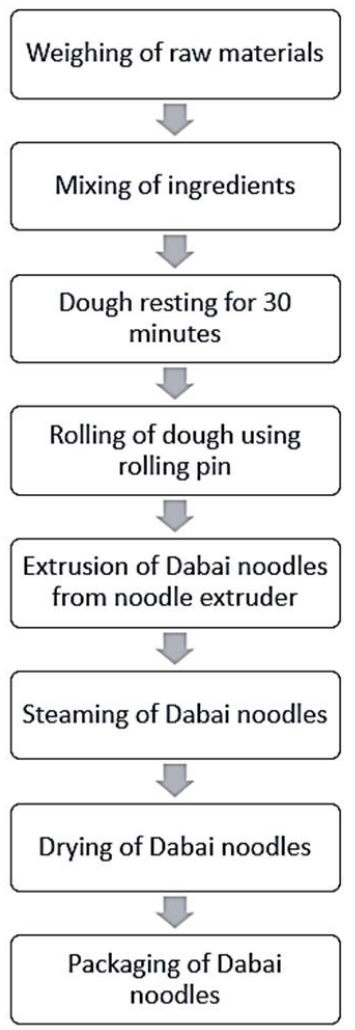

Figure 1: Preparation of Dabai noodles. Pakhare et.al, [9] 


\section{Sensory evaluation of Dabai noodles}

Dabai noodles $(250 \mathrm{~g}$ ) were cooked for 5 minutes in $1 \mathrm{~L}$ of salted water $(5 \% \mathrm{NaCI})$ and drained. Sensory tests were performed after 5 minutes of draining. Dabai noodles with four different formulations $(0 \%, 10 \%$, $20 \%$, and $30 \%$ Dabai) were evaluated. Total of 30 sensory evaluation panelists consist of both females and males were required to evaluate the taste, aroma, appearance, texture, overall acceptability, and purchase intention using 9-point hedonic scale.

Hedonic scale was used to capture the level of acceptance of consumers. By using hedonic scale, researchers get to know the information about the score of each attributes of food samples and hence helped in increasing the quality of products and consumer satisfaction. The product can be defined as product with quality if the mean liking score reach 7 or higher in the sensory evaluation. Besides, the strength and weakness of food samples with different formulae can clearly be seen for future improvement [10].

Panelists were allocated to comfortable individual booth for sensory evaluation to minimize visual distraction. $15 \mathrm{~g}$ of Dabai noodles were served in paper cup labelled with three-digit code to the panelists. The panelists were given scores of 1-9 ranging from 'Dislike extremely' to 'Like extremely' to determine the best proportion of Dabai powder to wheat flour in noodles composition. Analysis of Variance (ANOVA) was used to analyse the data obtained from the sensory evaluation [11].

\section{RESULTS AND DISCUSSION}

Figure 2 shows the radar chart of sensory evaluation of Dabai noodles. The radar chart was plotted based on data tabulated in Appendix 1. For colour section, Mauchly's Test of Sphericity was assumed $(\mathrm{p}>0.05)$. There was no significant different among the result for colour section as Tests of withinsubjects' effects (p.>0.05).

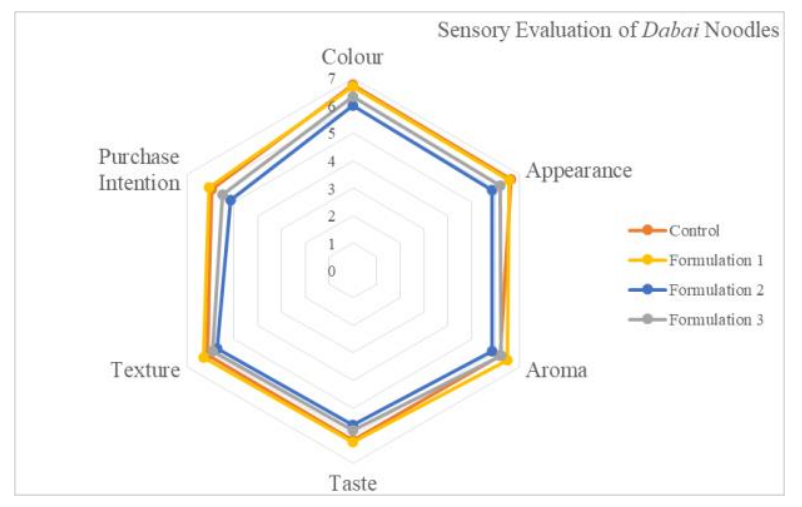

Figure 2: Sensory evaluation of Dabai noodles
The number 5 represent neither like or dislike, number 6 represent like slightly and number 7 represent like moderately. According to Figure 4.5, control has the highest mean of 6.77 followed by formulation 1 with mean of 6.70. Most of the panelists like the colour of control over formulation 1,2, and 3. Control has the whitest colour and the colour of Dabai noodles getting darker from formulation 1 to 3. Panelists has higher acceptance toward whiter noodles.

For appearance section, Mauchly's Test of Sphericity was not assumed $(p<0.05)$. GreenhouseGeisser is used as correction for sphericity not assumed. There was no significant different among the result for colour section as Tests of within-subjects' effects (p.>0.05). According to Figure 2, control has the highest mean of 6.67 followed by formulation 1 with mean of 6.60 .

Most of the panelists like the appearance of control over formulation 1,2, and 3. The appearance of control is smooth while the appearance of Dabai noodles getting rougher from formulation 1 to 3 . Panelists has higher acceptance toward smoother noodles.

For aroma section, Mauchly's Test of Sphericity was not assumed $(p<0.05)$. Greenhouse-Geisser is used as correction for sphericity not assumed. There was no significant different among the result for colour section as Tests of within-subjects' effects (p.>0.05). Referring to Figure 2, formulation 1 has the highest mean of 6.50 followed by formulation 3 with mean of 6.23 .

$80 \%$ of the panelists like the aroma of formulation 1 over control, formulation 2, and formulation 3 . Formulation 1 of Dabai noodles incorporated with lowest amount of Dabai among other formulation. Thus, panelists who familiar with Dabai fruits can accept the aroma of formulation 1. However, formulation 3 have the lowest score (5.87) because it contain higher level of fatty acid.

For taste section, Mauchly's Test of Sphericity was assumed ( $p>0.05)$. There was no significant different among the result for colour section as Tests of withinsubjects' effects (p.>0.05). According to Figure 4.5, formulation 1 has the highest mean of 6.23 followed by control with mean of 6.17.

Most of the panelists like the taste of formulation 1 over control, formulation 2, and formulation 3. From the feedback from the panelists, formulation 1 has higher score among the others because Dabai contribute to some flavour over the bland noodles while dislike the formulation 2 and 3 as they have stronger taste and more acidic.

For purchase intention section, Mauchly's Test of Sphericity was not assumed $(\mathrm{p}<0.05)$. GreenhouseGeisser is used as correction for sphericity not assumed. There was no significant different among the result for colour section as Tests of within-subjects' effects (p.>0.05). According to Figure 4.5, formulation 1 has 
the highest mean of 6.07 followed by control with mean of 5.93 .

Most of the panelists has higher purchase intention towards formulation 1 over control, formulation 2 , and formulation 3. Most of the panelists did not had high intention to buy Dabai noodles however they will choose Dabai noodles with formulation 1 if they have to make a choice.

Referring to Figure 3,71\% panelists like Dabai fruit while $30 \%$ panelists dislike Dabai fruit. Among those panelists who like Dabai fruit, $67 \%$ of them are male while $33 \%$ of them are female. Among those $30 \%$ panelists who dislike Dabai fruit, $67 \%$ of them are male while $33 \%$ of them are female. Besides, $90 \%$ panelists like noodles while $10 \%$ panelists dislike noodles. Among those panelists who like noodles, $67 \%$ of them are male while $33 \%$ of them are female.

Next, about $80 \%$ panelists would recommend Dabai noodles to others while $20 \%$ panelists would not recommend Dabai noodles to others. Among those $80 \%$ panelists who would recommend Dabai noodles to others, $67 \%$ of them are male while $33 \%$ of them are female. Among those $20 \%$ panelists who would not recommend Dabai noodles to others, $66 \%$ of them are male while $34 \%$ of them are female.

Dabai preferences among the panelists is around $50 \%$ as half of the male and female group like Dabai. Most of the panelists like to eat noodles and only a few of them dislike noodles. In the end of the sensory evaluation, most of the panelists would recommend Dabai noodles to others. About $25 \%$ of the male and female group would not recommend Dabai noodles to others.

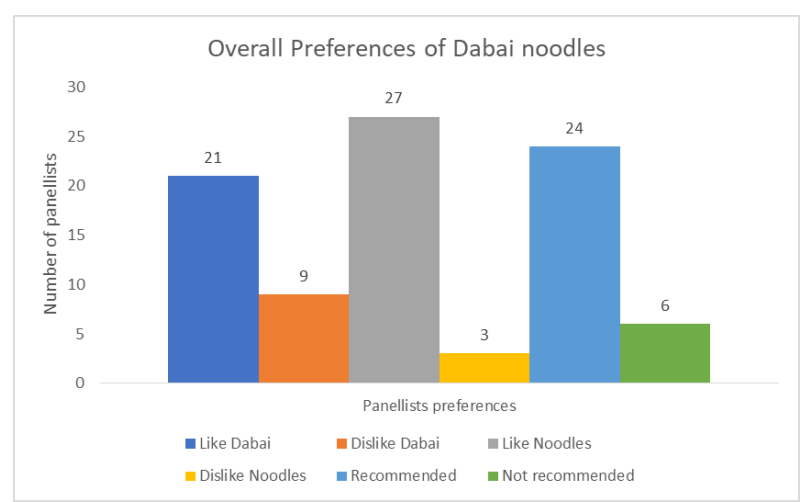

Figure 3: Overall Preferences of Dabai noodles

Discussing on Figure 4, 30\% panelists like control, 27\% panelists like Dabai noodles formulation 3 while $43 \%$ panelists like Dabai noodles formulation 1. Among those $30 \%$ panelists who like control, $67 \%$ of them are male while $33 \%$ of them are female. Among those panelists who like Dabai noodles formulation 3, $63 \%$ of them are male while $37 \%$ of them are female. Among those $43 \%$ panelists who like Dabai noodles formulation $1,69 \%$ of them are male while $31 \%$ of them are female. Dabai noodles formulation 1 has higher score when compared to control however there is no significant among them according to ANOVA. There is no single panelist recommend Dabai noodles formulation 2 to others. The reason behind this may due to the amount of Dabai powder incorporated with noodles. Dabai noodles formulation 2 contain $20 \%$ of Dabai powder. Thus, the intensity level may not being able to be detected in significant differences of formulation 2 and formulation 1.

Panelists are more likely to be attracted to slight increment $(10 \%)$ or bulky increment $(30 \%)$ of Dabai powder. Panelists may find that noodles incorporated with $10 \%$ Dabai powder are attractive for normal consumer while noodles incorporated with $30 \%$ Dabai powder are attractive and interesting for Dabai fruits lover. The higher percentages of Dabai powder lead to increasing likeness of noodles incorporated with Dabai powder.

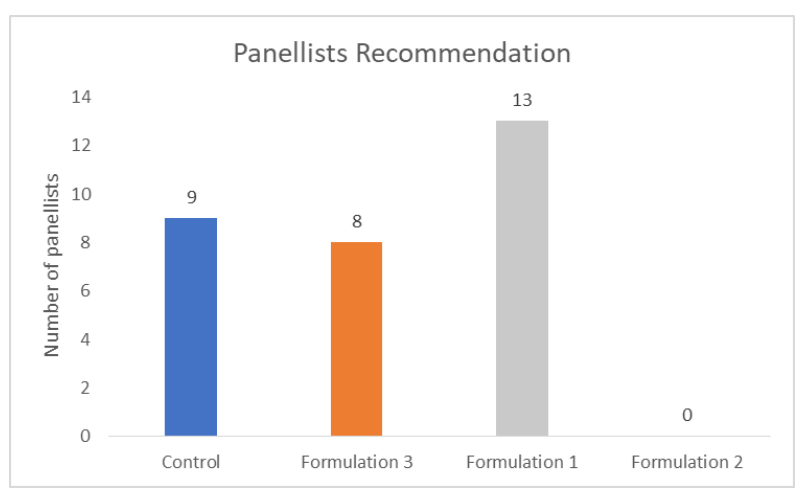

Figure 4: Panellists recommendation

\section{CONCLUSION}

The significance of this study is introducing the unique taste and aroma of Sarawak's indigenous fruit Dabai and produce new flavour noodles incorporated with Dabai powder. Thus, giving Dabai more opportunity to be commercialized in new food development area. Formulation 1 show the highest mean score in terms of overall acceptability and purchase intention. Microbial test, colour analysis, and phenolic compound analysis can be carried out to explore more about the shelf life and the nutritional value of Dabai noodles.

\section{ACKNOWLEDGEMENT}

Thanks are due to University of Technology Sarawak for financial support provided through the university's research grant (UCTS/RESEARCH/1/2019/5) and Universiti Putra Malaysia for the assistance throughout the study. The authors declare that there is no conflict of interest. 


\section{REFERENCES}

[1] Roach, J. (2005). 4,000-Year-Old Noodles Found in China. [Online] Available at: <https://www. nationalgeographic.com/news/2005/10/4-000-year -old-noodles-found-in-china/> [Accessed 29 May 2021].

[2] Zhang. N. and Ma. G. (2016). Noodles, Traditionally and today. Journal of Ethnic Foods, 3(3), pp. 209-212.

[3] Eyidemir. E. and Hayta. M. (2009). The effect of apricot kernel flour incorporation on the physicochemical and sensory properties of noodle. African Journal of Biotechnology, 8(1), pp. 85-80.

[4] Li. M., Dhital. S., and Wei. Y. (2017). Multilevel Structure of Wheat Starch and Its Relationship to Noodle Eating Qualities. Comprehensive Reviews in Food Science and Food Safety, 16(5), pp. 10421055.

[5] Lim, T. K. (2016). Canarium odontophyllum. In: Edible Medicinal and Non-Medicinal Plants. New York: Springer Netherlands, pp. 624-629.

[6] Xuan. T. C., and Azlan. A. (2016). Nutritional, Phytochemical and Pharmacological Properties of Canarium odontophyllum Miq. (Dabai) Fruit. PJSRR Pertanika Journal of Scholarly Research Reviews, 2(1), pp. 80-94.
[7] Armentrout, J. (2020). What We Mean By: Medium or Fine Sieve. [Online] Available at: $<$ https://www.finecooking.com/article/what-wemean-by-medium-or-fine-sieve $>$ [Accessed 29 December 2020].

[8] Adegunwa M. O., Bakare H. A., Akinola O. F. (2012). Enrichment of Noodles with Soy Flour and Carrot Powder. Nigerian Food Journal, 30(1), pp. 74-81.

[9] Pakhare K. N., Dagadkhair A. C., Udachan I. S., Andhale R. A. (2016). Studies on Preparation and Quality of Nutritious Noodles by Incorporation of Defatted Rice Bran and Soy Flour. Journal of Food Processing \& Technology, 7(10), pp. 1-4.

[10] Everitt, M. (2009). Global Issues in Food Science and Technology. In: A. M. D. L. W. S. K. B. a. P. C. Gustavo Barbosa-Cánovas, ed. Consumer-Targeted Sensory Quality. New York: Academic Press, pp. 117-128.

[11] Fari M. J. M., Rajapaksa. D., Ranaweera. K. K. D. S. (2011). Quality characteristics of noodles made from selected varieties of Sri Lankan rice with different physicochemical characteristics. Journal of the National Science Foundation of Sri Lanka, 39(1), pp. 53-60. 